\title{
Efficient Everolimus Treatment for Metastatic Castration Resistant Prostate Cancer with AKTI Mutation: A Case Report
}

\author{
Zhe $\mathrm{Yu}^{\mathrm{l}, *}$ \\ Wei Wei ${ }^{1} *$ \\ Hongruo Liu' \\ Evenki Pan ${ }^{2}$ \\ Peng Yang ${ }^{2}$ \\ Kui Jiang' \\ 'Department of Medical Oncology, The \\ Second Affiliated Hospital of Dalian \\ Medical University, Dalian, Liaoning, \\ People's Republic of China; ${ }^{2}$ Nanjing \\ Genesseq Technology Inc., Nanjing, \\ Jiangsu, People's Republic of China \\ *These authors contributed equally to \\ this work
}

Correspondence: Kui Jiang

Department of Medical Oncology, The

Second Affiliated Hospital of Dalian

Medical University, Dalian, Liaoning,

People's Republic of China

Email jk04II@I63.com

\begin{abstract}
Metastatic castration resistant prostate cancer (mCRPC), the advanced stage of prostate cancer (PCa), develops resistance to first line androgen deprivation therapy (ADT). Aberrant androgen receptor (AR) and PI3K-Akt-mTOR signaling pathway are responsible for the development and progression of MCRPC. We herein describe a case of a 64-year-old male mCRPC patient with somatic $A K T 1$ and $A R$ mutations. The patient, who had been heavily pretreated by ADT and AR inhibitors, showed stable disease progression when he received everolimus, an mTOR inhibitor. The PSA level dropped drastically from $1493.0 \mathrm{ng} /$ $\mathrm{mL}$ to $237.6 \mathrm{ng} / \mathrm{mL}$, after 3 months of treatment. The overall survival (OS) was 43 months, of which the progression-free survival (PFS) with everolimus treatment was 7 months. The administration of mTOR inhibitor, everolimus, could achieve good clinical responses along with prolonging PFS for mCRPC patients harboring AKT1 mutations. Technology in precision medicine, such as targeted next-generation sequencing (NGS) of cancer-relevant genes, has promising function in personalized therapy.
\end{abstract}

Keywords: castration resistant prostate cancer, androgen receptor, everolimus, nextgeneration sequencing

\section{Introduction}

Prostate cancer (PCa), an epithelial malignant tumor developing in the prostate, is the second most common malignant tumor among men in the world. ${ }^{1}$ Androgen deprivation therapy (ADT) via chemical or surgical castration, as the first-line treatment for metastatic $\mathrm{PCa}$, can temporarily achieve good clinical responses. However, subsequent androgen deprivation resistance is widely observed in metastatic PCa patients receiving $\mathrm{ADT}$, resulting in metastasis castration-resistant prostate cancer (mCRPC). ${ }^{2}$ A combination of mutated genes (such as TP53, RB1, PTEN, and $B R C A 1 / 2$ ), activated signaling pathways (such as PI3K/AKT/mTOR, WNT/ $\beta$ catenin, and SRC), and other mechanisms are involved in the progression and evolution of PCa. ${ }^{3}$ A majority of castration resistant prostate cancer (CRPC) patients are characterized with mutations and copy number alterations of genes related to the PI3KAkt-mTOR signaling pathway, ${ }^{4}$ which plays a crucial role in castration-resistance and CRPC development. ${ }^{5}$ Clinical trials have been launched to investigate whether mTOR, pan/selective-PI3K and Akt inhibitors are novel targeted therapy agents of mCRPC. ${ }^{6}$ Everolimus (RAD001), an mTOR inhibitor, can effectively limit tumor growth via inhibiting cell proliferation, angiogenesis, and tumor cell autophagy. ${ }^{7}$ Food and Drug 
Administration (FDA) has approved the application of everolimus in advanced renal cell carcinoma, breast cancer, and other tumors. ${ }^{8}$ However, few case reports have clarified its role in $\mathrm{mCRPC}$. Herein, we report a case of everolimus treatment against mCRPC in a patient with $A K T 1$ and $A R$ mutations, to provide $\mathrm{mCRPC}$ patients with an alternative treatment option.

\section{Case Presentation}

A 64-year-old man with a 2-year history of dysuria presented increased dysuria accompanying pain in his left leg. He was diagnosed as PCa with a high Gleason score (5 plus 3) in April 2016, by undergoing pathological tissue biopsy. His serum prostate-specific antigen (PSA) was over $100 \mathrm{ng} / \mathrm{mL}$, and emission computed tomography (ECT) imaging showed systemic bone metastasis. Throughout the treatment period, we monitored the PSA level to evaluate the therapeutic effect (Figure 1). Prostatectomy was performed followed by 4-month flutamide (250 $\mathrm{mg}$ three times a day), continued goserelin (3.6 mg every 4 weeks until passing away), and 6-week chemotherapy with docetaxel $\left(75 \mathrm{mg} / \mathrm{m}^{2}\right.$ every 3 weeks $)$ combined with zoledronic acid therapy (4 mg every 4 weeks until month 36). At the end of month 4, PSA level declined from $125.20 \mathrm{ng} / \mathrm{mL}$ to $0.02 \mathrm{ng} / \mathrm{mL}$, and the tumor size of the lung metastasis also decreased from the whole lung to a small lesion; however, the testosterone remained at castrate level throughout the drug treatment period. From month 8 to month 14 , the patient was treated with bicalutamide (50 $\mathrm{mg}$ once a day) as an antiandrogen drug, and the PSA level rose gradually from $0.63 \mathrm{ng} / \mathrm{mL}$ to $14.51 \mathrm{ng} / \mathrm{mL}$. To treat against the increasing PSA, the patient received a 9-month combination treatment of abiraterone (1 g once a day) and prednisone ( $5 \mathrm{mg}$ twice a day), during which the PSA level finally increased to $26.64 \mathrm{ng} / \mathrm{mL}$, despite the temporary decline to $15.00 \mathrm{ng} / \mathrm{mL}$. Although we measured a transient decrease of PSA in this period, no appreciable improvement was observed in pulmonary pathology imaging.

The patient was then treated with docetaxel $\left(75 \mathrm{mg} / \mathrm{m}^{2}\right)$ plus prednisone ( $5 \mathrm{mg}$ twice a day) for 5 months, carboplatin (area under the curve: 5) for 3 months, and enzalutamide (160 mg once a day) for 3 months, sequentially. The metastasis in lungs and bone kept progressing, accompanying with the increasing PSA level which reached $45.53 \mathrm{ng} / \mathrm{mL}, 92.07 \mathrm{ng} / \mathrm{mL}$, and $169.6 \mathrm{ng} / \mathrm{mL}$, respectively, after completing each of these three treatments. Based on these poor responses, olaparib (300 mg twice a day) combined with abiraterone (1 g once a day) was given to the patient for 4 months; however, PSA peaked at $1,493.0 \mathrm{ng} / \mathrm{mL}$. Thus, tumor tissue biopsy of the right lung was sampled and performed with immunohistochemistry. Due to the positive staining results of both PSA and prostate specific membrane antigen (PSMA), we found this adenocarcinoma sample in the lung was derived from PCa. To clarify the genomic profile of tumor tissue,

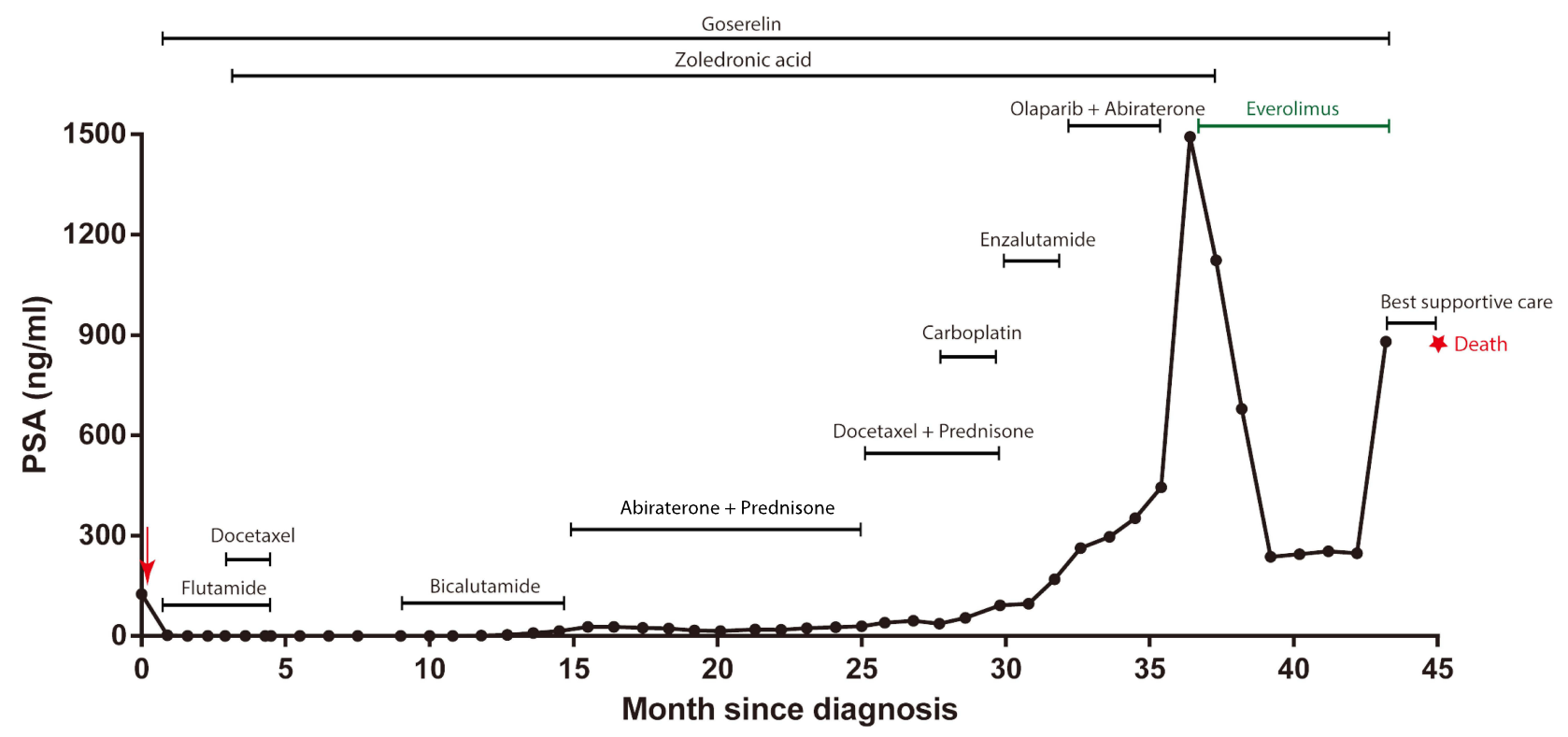

Figure I The surveillance of PSA level since initial diagnosis. The red arrow represents the prostatectomy, and the red star represents death. The online part and the line length represent the therapeutic regimen and therapy time, respectively. The PSA level at death was unknown. 
the lung metastasis and plasma samples underwent NGS analysis (Nanjing Geneseeq Technology Inc., Nanjing, Jiangsu, China) for 425 cancer-relevant genes. AKT1 mutation was observed in both tissue and plasma samples, while $A R$ mutation was only detected in the tissue sample (Figure 2 and Supplementary Table 1). According to the sequencing result, oral administration of everolimus (10 mg once daily) was started and continued until passing away. After receiving this $A K T 1$ mutation targeted treatment for 3 months, the PSA level drastically dropped from $1,493.0 \mathrm{ng} / \mathrm{mL}$, the highest PSA level, to $237.6 \mathrm{ng} / \mathrm{mL}$. CT imaging also revealed that the size of the lung lesion was remarkably reduced, and the size of the bone metastasis remained stable (Figure 3). In the following 3 months, PSA level remained stable, under $300 \mathrm{ng} / \mathrm{mL}$, followed by leaping to $880.3 \mathrm{ng} / \mathrm{mL}$. Unfortunately, the patient died in January 2020. Supplementary Table 2 summarizes the dosage and frequency of medication.

\section{Discussion}

ADT is the internationally recognized standard treatment for PCa. ${ }^{9}$ After 18 24 months of ADT treatment, most

A

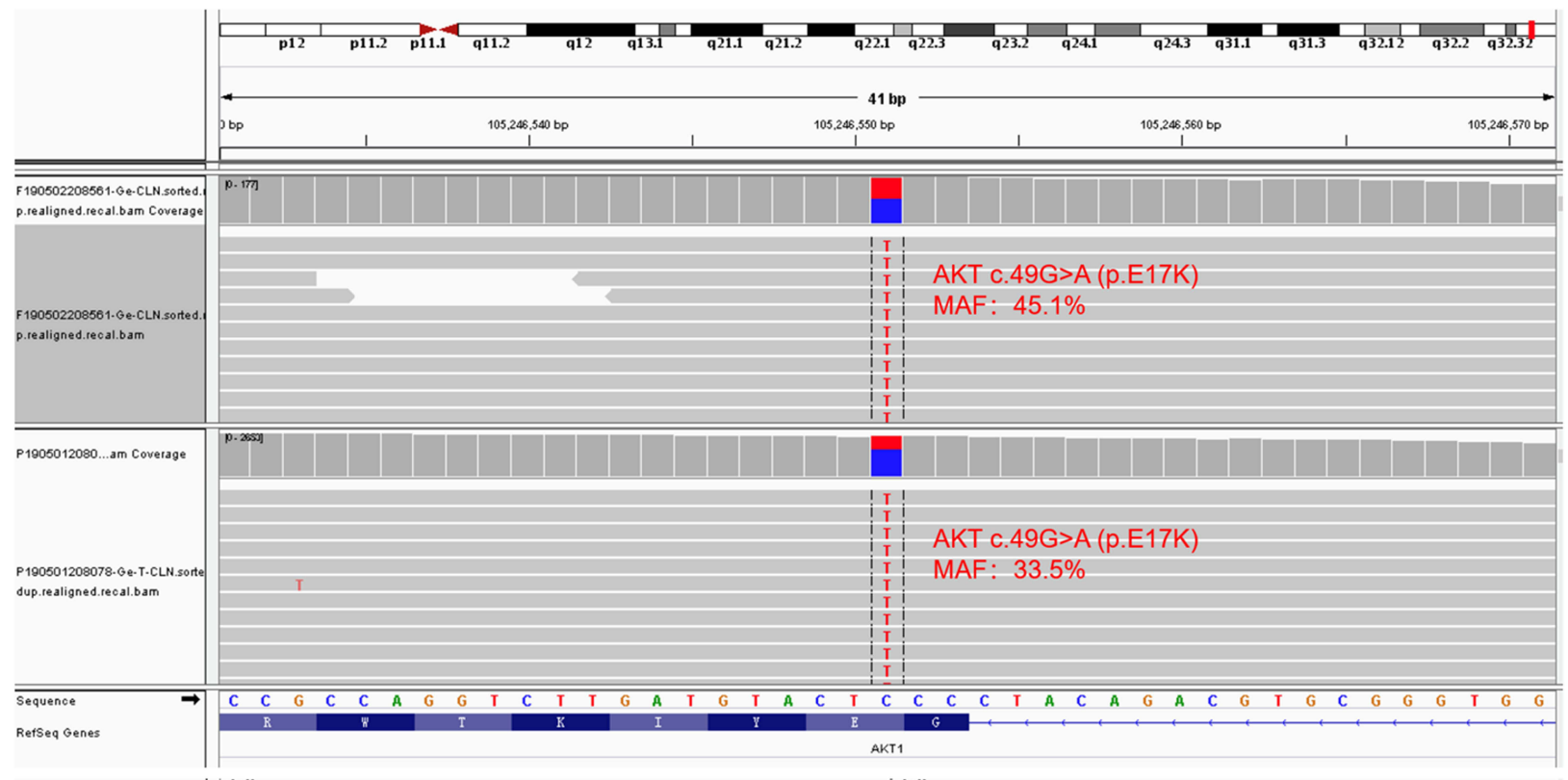

B

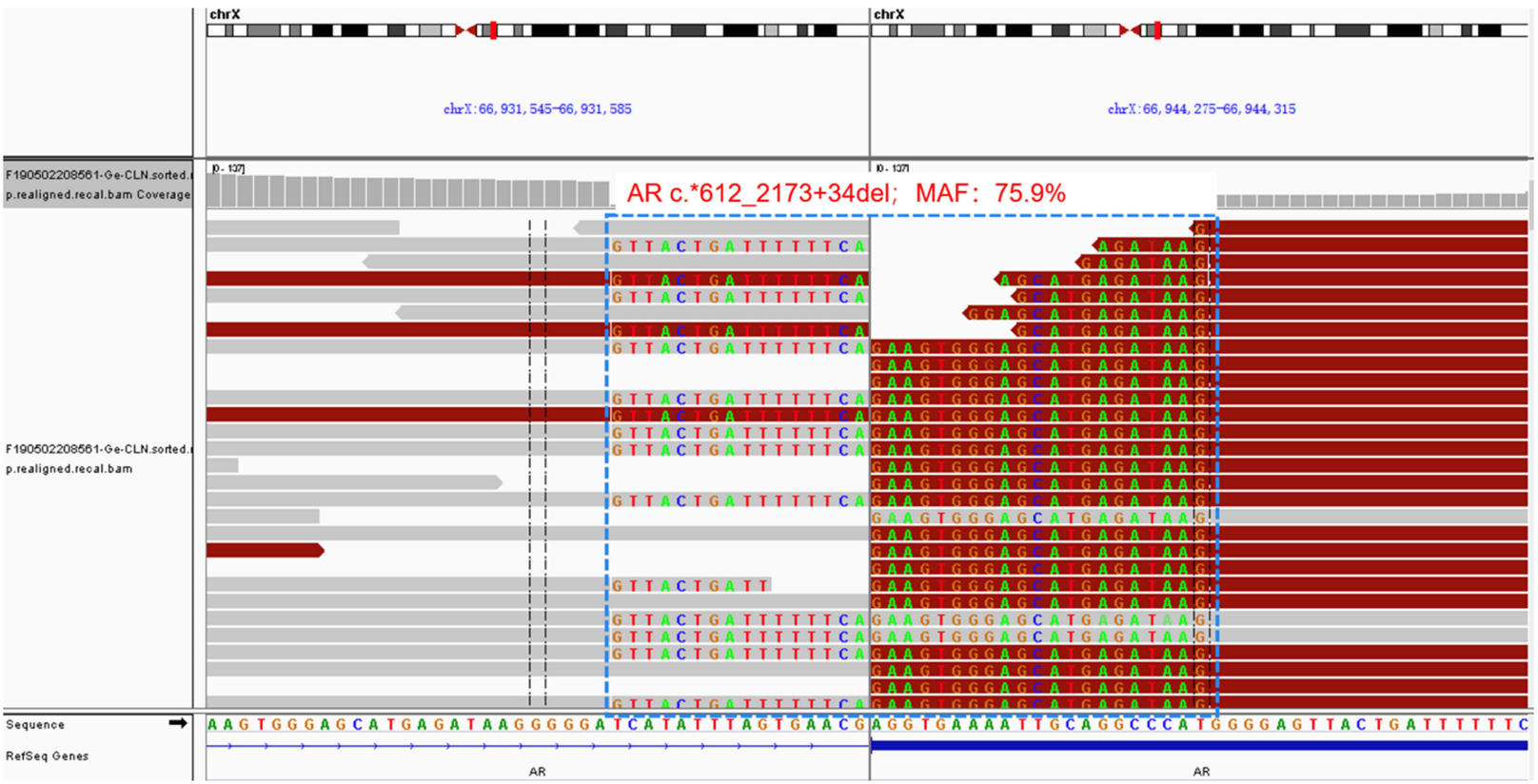

Figure 2 NGS analysis of the lung metastasis and plasma samples. (A) Missense mutation of AKTI p.EI7K in lung tissue and plasma samples. (B) The deletion mutation (c*612-2173+34del) of exons 5-7 and a part of exon 8 of $A R$ in lung tissue. 


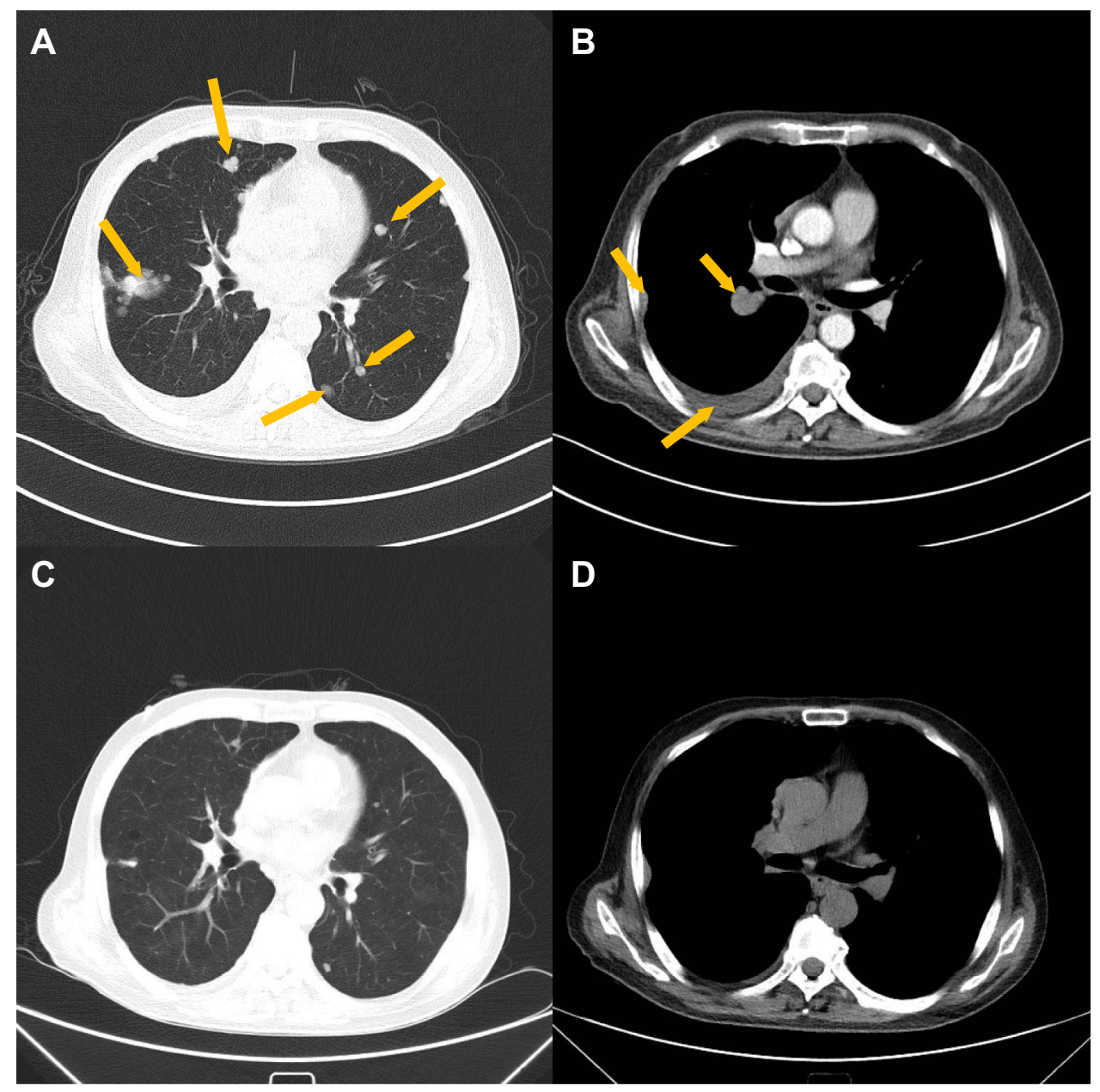

Figure 3 The lung and pleural metastasis were reduced after everolimus treatment. (A) CT image of lung metastasis before medication of everolimus. Arrows represent pulmonary metastases. (B) CT image of pleural metastasis before medication of everolimus. The arrow in the lower left corner represents the pleural effusion, and other arrows represent pulmonary metastases. (C) CT image of lung metastasis after everolimus treatment for 3 months. (D) CT image of pleural metastasis after everolimus treatment for 3 months.

PCa cases irreversibly develop into mCPRC, of which the median survival time is around $1 \sim 2$ years. ${ }^{10}$ Herein, we reported the treatment process of a mCRPC patient with $A K T 1$ and $A R$ mutations and revealed that everolimus was a potential option of limiting $\mathrm{mCRPC}$ progression.

This mCRPC patient successively experienced multiple approved therapies, including docetaxel, abiraterone, and enzalutamide. However, all of them yielded to unstoppable degenerative disease. Genomic profiling, using NGS, detected $A R$ deletion and $A K T 1$ (E17K) mutation in metastatic lung tumor tissue and plasma samples. An aberrant AR signaling pathway is widely considered as a dominant driver of $\mathrm{PCa} .{ }^{11}$ Increased expression of androgen-synthesizing enzymes continuously sustained elevated androgen levels and contributed to $A R$ activation. $^{12}$ The detected AR deletion c.*612_2173 +34del included exons 5-7 and a part of exon 8 that code for the ligand binding domain interacting with dihydrotestosterone. This deletion was very likely to be associated with $A R$ splice variant $(A R-V)$ of $A R^{\text {v567es }}$ or ARV12. ${ }^{13}$ These two types of AR-Vs were considered to be constitutively active and contribute to ADT resistance. ${ }^{14}$ Additionally, AR-Vs could not interact with heat shock protein or combine with androgen response elements in the nucleus to regular PSA expression. ${ }^{15}$ According to previous studies, ${ }^{16}$ we deduced that bicalutamide resistance was attributable to AR-Vs. Moreover, various clinical studies found that abiraterone and enzalutamide had no satisfactory long-term therapeutic effect in the mCRPC patients with AR-Vs. ${ }^{17}$ These findings could explain the high-level PSA of the patient, especially for the late stage of treatment. It was also notable that the PI3K-AKT-mTOR signaling pathway was altered in almost $100 \%$ of advanced-stage PCas. ${ }^{18}$ Many studies 
have revealed the abnormal activities of AKT and mTOR proteins in prostate cancer tissue, which implies the crucial role of PI3K-AKT-mTOR in the occurrence and development of PCa. ${ }^{19,20}$ The crosstalk signaling between $\mathrm{AR}$ and PI3K-AKT-mTOR pathways is hyperactive in mCRPC. Hence, the inhibitors of the above two pathways would produce a promising therapeutic effect in mCRPC.

Of note, $A K T 1(\mathrm{E} 17 \mathrm{~K})$ mutations stimulate downstream signals of PI3K-AKT-mTOR that cause tumor cells to emerge transformed. ${ }^{21}$ Also, AKT1 (E17K) mutant oncoproteins can selectively destroy rare, quiescent, chemotherapy-resistant, and tumor-promoting $\mathrm{AKT} 1^{\text {low }}$ quiescent cancer cells (QCC). ${ }^{22}$ Multiple investigations have reported remarkably longer survival time after everolimus treatment in patients carrying $A K T 1$ (E17K) mutations. ${ }^{23}$ Hence, we adopted everolimus to halt disease progression in this case with ADT treatment resistance. The effectiveness of everolimus could be proved by the drop of PSA level and the reduced size of lung lesion. The overall survival (OS) of this patient was 43 months, including the 7-month progression free survival (PFS) with everolimus. The patient's OS was longer than the median OS (around 20 months) of mCRPC patient with no effective treatments. ${ }^{24,25}$ Such evidence indicated everolimus could effectively alleviate mCRPC degeneration. Some Phase II clinical trials showed that mCRPC patients did not benefit from everolimus; ${ }^{26,27}$ however, patients in these studies did not undergo genomic profiling and their $A K T$ mutation status was unknown. Thus, our case suggested that either tumor tissue or plasma samples of mCRPC patients should be performed with comprehensive genomic profiling before being treated with targeted therapy such as everolimus.

In summary, the case report complemented the clinical application of everolimus against mCRPC. The sharply decreased PSA level was very likely to be associated with the administration of everolimus in this mCRPC patient harboring $A R$ and $A K T 1$ E17K mutations. He also achieved relatively good responses to everolimus, including reduced or stable tumor size of metastases, and longer PFS of 7 months. Clinical trials, enrolling patients with mutated PI3K-AKT-mTOR and AR signaling pathways, should be launched to further investigate the efficacy of everolimus in $\mathrm{mCRPC}$.

\section{Abbreviations}

$\mathrm{PCa}$, prostate cancer; CRPC, castration resistant prostate cancer; mCRPC, metastatic castration resistant prostate cancer; ADT, androgen deprivation therapy; AR, androgen receptor;
OS, overall survival; PFS, progression-free survival; NGS, next-generation sequencing; FDA, Food and Drug Administration; PSA, prostate-specific antigen; ECT, emission computed tomography; PSMA, prostate specific membrane antigen; AR-Vs, AR splice variants; QCC, quiescent cancer cells.

\section{Data Sharing Statement}

Additional data and materials related to the genetic tests, pathologic reports, treatment information, and images are available for review upon reasonable request.

\section{Ethics Approval and Consent to Participate}

This research was approved by the ethics committee of the Second Affiliated Hospital of Dalian Medical University. Written informed consent for publication of the clinical details and images was obtained from the patient and wasn't required institutional approval.

\section{Acknowledgments}

We sincerely thank the patient for supporting our work.

\section{Author Contributions}

$\mathrm{ZY}$ and WW contributed equally to the work. All authors made a significant contribution to the work reported, whether that is in the conception, study design, execution, acquisition of data, analysis and interpretation, or in all these areas; took part in drafting, revising, or critically reviewing the article; gave final approval of the version to be published; have agreed on the journal to which the article has been submitted; and agree to be accountable for all aspects of the work.

\section{Disclosure}

Evenki Pan and Peng Yang are employed by Nanjing Geneseeq Technology Inc., China. The remaining authors declare that the research was conducted in the absence of any commercial or financial relationships that could be construed as a potential conflict of interest.

\section{References}

1. Sternberg CN, Fizazi K, Saad F, et al. Enzalutamide and survival in nonmetastatic, castration-resistant prostate cancer. $N$ Engl J Med. 2020;382(23):2197-2206.

2. Saad F, Cella D, Basch E, et al. Effect of apalutamide on health-related quality of life in patients with non-metastatic castration-resistant prostate cancer: an analysis of the SPARTAN randomised, placebo-controlled, Phase 3 trial. Lancet Oncol. 2018;19(10): 1404-1416. 
3. Tong D. Unravelling the molecular mechanisms of prostate cancer evolution from genotype to phenotype. Crit Rev Oncol Hematol. 2021;27:103370.

4. Ciccarese C, Massari F, Iacovelli R, et al. Prostate cancer heterogeneity: discovering novel molecular targets for therapy. Cancer Treat Rev. 2017;54:68-73.

5. Edlind MP, Hsieh AC. PI3K-AKT-mTOR signaling in prostate cancer progression and androgen deprivation therapy resistance. Asian $J$ Androl. 2014;16(3):378-386.

6. Cham J, Venkateswaran AR, Bhangoo M. Targeting the PI3K-AKTmTOR pathway in castration resistant prostate cancer: a review article. Clin Genitourin Cancer. 2021. doi:10.1530/ERC-12-0394

7. Doi T, Muro K, Boku N, et al. Multicenter phase II study of everolimus in patients with previously treated metastatic gastric cancer. J Clin Oncol. 2010;28(11):1904-1910.

8. Gabardi S, Baroletti SA. Everolimus: a proliferation signal inhibitor with clinical applications in organ transplantation, oncology, and cardiology. Pharmacotherapy. 2010;30(10):1044-1056.

9. Nelson WG, Haffner MC, Yegnasubramanian S. Beefing up prostate cancer therapy with performance-enhancing (anti-) steroids. Cancer Cell. 2011;20(1):7-9.

10. Shigeta K, Kosaka T, Hongo H, et al. Castration-resistant prostate cancer patients who had poor response on first androgen deprivation therapy would obtain certain clinical benefit from early docetaxel administration. Int J Clin Oncol. 2019;24(5):546-553.

11. Coutinho I, Day TK, Tilley WD, Selth LA. Androgen receptor signaling in castration-resistant prostate cancer: a lesson in persistence. Endocr Relat Cancer. 2016;23(12):T179-t197.

12. Yuan X, Cai C, Chen S, Chen S, Yu Z, Balk SP. Androgen receptor functions in castration-resistant prostate cancer and mechanisms of resistance to new agents targeting the androgen axis. Oncogene. 2014;33(22):2815-2825.

13. Lu C, Luo J. Decoding the androgen receptor splice variants. Transl Androl Urol. 2013;2(3):178.

14. Wadosky KM, Koochekpour S. Androgen receptor splice variants and prostate cancer: from bench to bedside. Oncotarget. 2017;8(11):18550.

15. Zoubeidi A, Zardan A, Beraldi E, et al. Cooperative interactions between androgen receptor (AR) and heat-shock protein 27 facilitate AR transcriptional activity. Cancer Res. 2007;67(21):10455-10465.
16. Dalal K, Ban F, Li H, et al. Selectively targeting the dimerization interface of human androgen receptor with small-molecules to treat castration-resistant prostate cancer. Cancer Lett. 2018;437:35-43.

17. Nadal R, Bellmunt J. The evolving role of enzalutamide on the treatment of prostate cancer. Future Oncol. 2016;12(5):607-616.

18. Xu F, Na L, Li Y, Chen L. Roles of the PI3K/AKT/mTOR signalling pathways in neurodegenerative diseases and tumours. Cell Biosci. 2020;10:54

19. Bitting RL, Armstrong AJ. Targeting the PI $3 \mathrm{~K} / \mathrm{Akt} / \mathrm{mTOR}$ pathway in castration-resistant prostate cancer. Endocr Relat Cancer. 2013;20 (3):R83-R99.

20. Grünwald V, DeGraffenried L, Russel D, Friedrichs WE, Ray RB, Hidalgo M. Inhibitors of mTOR reverse doxorubicin resistance conferred by PTEN status in prostate cancer cells. Cancer Res. 2002;62 (21):6141-6145

21. Chen Y, Huang L, Dong Y, et al. Effect of AKT1 (p. E17K) hotspot mutation on malignant tumorigenesis and prognosis. Front Cell Develop Biol. 2020;8:573599.

22. Alves CP, Dey-Guha I, Kabraji S, et al. AKT1(low) quiescent cancer cells promote solid tumor growth. Mol Cancer Ther. 2018;17 (1):254-263.

23. Omarini C, Filieri ME, Bettelli S, et al. Mutational profile of metastatic breast cancer tissue in patients treated with exemestane plus everolimus. Biomed Res Int. 2018;2018:3756981.

24. Fizazi K, Scher HI, Molina A, et al. Abiraterone acetate for treatment of metastatic castration-resistant prostate cancer: final overall survival analysis of the COU-AA-301 randomised, double-blind, placebo-controlled phase 3 study. Lancet Oncol. 2012;13 (10):983-992.

25. George DJ, Sartor O, Miller K, et al. Treatment patterns and outcomes in patients with metastatic castration-resistant prostate cancer in a real-world clinical practice setting in the United States. Clin Genitourin Cancer. 2020;18(4):284-294.

26. Koshkin VS, Mir MC, Barata P, et al. Randomized phase II trial of neoadjuvant everolimus in patients with high-risk localized prostate cancer. Invest New Drugs. 2019;37(3):559-566.

27. George DJ, Halabi S, Healy P, et al. Phase 2 clinical trial of TORC1 inhibition with everolimus in men with metastatic castration-resistant prostate cancer. Urol Oncol. 2020;38(3):79.e15-79.e22.
OncoTargets and Therapy

\section{Publish your work in this journal}

OncoTargets and Therapy is an international, peer-reviewed, open access journal focusing on the pathological basis of all cancers, potential targets for therapy and treatment protocols employed to improve the management of cancer patients. The journal also focuses on the impact of management programs and new therapeutic agents and protocols on patient perspectives such as quality of life, adherence and satisfaction. The manuscript management system is completely online and includes a very quick and fair peer-review system, which is all easy to use. Visit http://www.dovepress.com/ testimonials.php to read real quotes from published authors. 